\title{
Two Subsets of HLA-DQA1 Alleles Mark Phenotypic Variation in Levels of Insulin Autoantibodies in First Degree Relatives at Risk for Insulin-dependent Diabetes
}

\author{
Alberto Pugliese, * Teodorica Bugawan," Rocio Moromisato, ${ }^{\star}$ Zuheir L. Awdeh, ${ }^{\star}$ Chester A. Alper, ${ }^{\star}$ Richard A. Jackson, \\ Henry A. Erlich," and George S. Eisenbarth * \\ * Barbara Davis Center for Childhood Diabetes, University of Colorado Health Sciences Center, Denver, Colorado 80262; ${ }^{\ddagger}$ The Center for \\ Blood Research, Harvard Medical School, Boston, Massachusetts 02115; ${ }^{\S}$ Joslin Diabetes Center, Harvard Medical School, Boston, \\ Massachusetts 02215; and "Human Genetics Department, Roche Molecular Systems, Alameda, California 94501
}

\begin{abstract}
Levels of insulin autoantibodies (IAA) vary among different first degree relatives of insulin-dependent diabetes mellitus patients, suggesting genetic regulation. We previously reported elevated IAA among DR4-positive at risk relatives. In this study, $72 / 82$ at risk relatives were IAA positive, of whom $75 \%$ (54/72) carried DR4 versus $20 \%(2 / 10)$ of IAA-negative relatives $(P=0.0004)$. However, 69\% $(18 / 26)$ of DR4-negative relatives were IAA positive. Since DR4 did not account for all IAA positivity, we analyzed DQA1 and DQB1 alleles. Homozygosity for DQA1 alleles deriving from the evolutionary lineage 4 (*0401, *0501, *0601) was associated with low IAA levels, while lineage 1-3 alleles ( $\star_{0101}, \star_{0102,}^{*} 0103,{ }^{*} 0201,{ }^{*} 0301$ ) correlated with higher levels. Most $(93 \%, 65 / 70)$ relatives with lineage 1-3 alleles were IAA positive (mean $=360 \pm 63$ $\mathrm{SEM} \mathrm{nU/ml).} \mathrm{Only} \mathrm{7/12} \mathrm{relatives} \mathrm{homozygous} \mathrm{for} \mathrm{lineage} 4$ alleles were IAA-positive, with lower levels than relatives with lineage 1-3 alleles $($ mean $=55 \pm 15 \mathrm{SEM} \mathrm{nU} / \mathrm{ml}, P<0.0001$; $7 / 12$ vs $65 / 70, P=0.004)$. The amino acid sequences of lineage 1-3 alleles uniquely share glutamic acid ( $E$ ) and phenylalanine (F) at positions 40 and 51 (EF alleles). Lineage 4 alleles have glycine (G) and leucine (L) at those positions (GL alleles $) .90 \%(65 / 72)$ of IAA-positive relatives had an EF allele, while only $75 \%(54 / 72)$ had DR4 $(P=0.01)$. Homozygosity for GL alleles (often DQA1 * 0501 on DR3 haplotypes) correlated with little or no humoral response to insulin. Thus, HLADQB1 GL alleles, or other genes on haplotypes (e.g., DR3) that carry these DQA1 alleles, may confer recessive low responsiveness to insulin. (J. Clin. Invest. 1994. 93:2447-2452.) Key words: autoimmunity - diabetes mellitus, insulin-dependent • insulin autoantibodies - HLA class II antigens - HLA-DQA1 antigens
\end{abstract}

\section{Introduction}

Type I diabetes (IDDM) ${ }^{1}$ is considered a chronic autoimmune disease resulting in the destruction of insulin-producing cells in the pancreas (1-3). Several islet molecules are targeted during

Address correspondence to George S. Eisenbarth, M.D., Ph.D., Barbara Davis Center for Childhood Diabetes, University of Colorado, Health Sciences Center, 4200 East 9th Avenue, Box B140, Denver, CO 80262.

Received for publication 3 August 1993 and in revised form 13 December 1993.

1. Abbreviations used in this paper: C. I., confidence interval; DQA1 EF, lineage 1-3 DQA 1 alleles; DQA 1 GL, lineage 4 DQA 1 alleles; IAA, insulin autoantibodies; ICA, islet cell antibodies; IDDM, insulin-dependent diabetes mellitus; SSO, sequence-specific oligonucleotide; TMAC, tetramethylammonium chloride.

J. Clin. Invest.

(C) The American Society for Clinical Investigation, Inc. $0021-9738 / 94 / 06 / 2447 / 06 \$ 2.00$

Volume 93, June 1994, 2447-2452 the autoimmune response, and autoantibodies against these molecules are detectable in the sera of most prediabetics or newly diagnosed IDDM patients $(1,2)$. It is still not known if there is a precise pattern of humoral autoimmunity leading to IDDM, and indeed we are learning that different individuals may have a different combination of autoantibodies with a different order of appearance during the prediabetic period. The heterogeneity of the autoimmune process may be in part determined by genetic factors.

Among the target autoantigens, insulin is the only $\beta$ cellspecific autoantigen identified to date, and thus immune responsiveness to this molecule may have an important role in the autoimmune process $(4,5)$. We have reported an inverse log-linear correlation between insulin autoantibody (IAA) levels and the age of diabetes onset, with the highest levels found in children who became diabetic before age 5 (6). Autoantibody-positive relatives of IDDM patients have different levels of IAA. Individual levels of IAA are often stable over long periods of time, changing very little during the prediabetic period (7). These observations suggest that the extent of the immune response to insulin may be at least in part genetically determined. Indeed, we have previously reported a significant association of high levels of IAA with HLA-DR4 haplotypes (8). However, DR4 did not account for all the individuals with high IAA levels.

The aim of our study was to further characterize HLA alleles associated with the humoral response to insulin during the prediabetic period in 82 first degree relatives at risk for IDDM, who were defined at risk if they were either IAA or islet cell antibody (ICA) positive or because they developed IDDM on follow-up regardless of their autoantibody status.

We find evidence that two different subsets of DQA 1 alleles derived from evolutionary lineages $1-3$ and $4(9,10)$ are associated with phenotypic variation in levels of IAA. In particular, homozygosity for lineage 4 alleles (including DQA $1{ }^{*} 0501$ found on DR3 and DR5 haplotypes, as well as the much rarer ${ }^{*} 0401$ and ${ }^{*} 0601$ ) is associated with low levels of IAA. In contrast, lineage 1-3 DQA 1 alleles (including DQA $1 * 0301$ found on DR4 haplotypes) are associated with high levels of IAA. Of note, only lineage 1-3 DQA 1 alleles carry glutamic acid at position 40 and phenylalanine at position 51 of their second exon. Therefore, the presence of these residues is shared only by those DQA1 alleles associated with high IAA levels both on DR4 and non-DR4 haplotypes. Our data lead us to speculate that these DQA1 sequences shared by lineage 1-3 alleles may facilitate the presentation of insulin to the immune system and determine higher levels of autoantibodies once tolerance to insulin is broken during the prediabetic period.

\section{Methods}

$I A A$ and ICA assays. IAA was measured by a competitive radioimmunoassay as described previously (6). Our upper limit of normal is 39 $\mathrm{nU} / \mathrm{ml}$ ( $3 \mathrm{SD}$ above normal). Reported IAA values represent the indi- 
vidual mean level of several autoantibody measurements before insulin therapy. ICA was determined as reported previously (11) including the restricted and nonrestricted subtypes (12). According to this classification, relatives with restricted ICA have a lower risk of diabetes compared with relatives with nonrestricted ICA. However, three relatives with restricted ICA were included in this analysis, since one of them became diabetic on follow-up at age 68 , and the other two had elevated IAA levels.

Subjects. After cytoplasmic ICA and IAA screening of $\sim 6,000$ first degree relatives of IDDM patients, we have analyzed the HLA type and IAA level of 82 individuals at increased risk for IDDM. Subjects were defined at risk for IDDM if they had one or a combination of the following: ( $a$ ) they had nonrestricted ICA $>20$ JDF units; $(b)$ they had been followed to the development of overt IDDM; and $(c)$ they had a mean IAA level $>39 \mathrm{nU} / \mathrm{ml}$ in the absence of other antiislet autoantibodies.

Table I illustrates the autoantibody status of the above 82 relatives subdivided by progression to overt diabetes. 72 of 82 relatives had IAA levels $>39 \mathrm{nU} / \mathrm{ml}$, of which 40 were ICA positive, and 32 were ICA negative. 49 relatives were ICA positive ( 46 had nonrestricted ICA, and 3 had restricted ICA). Of the $34(41.4 \%)$ relatives who became diabetic on follow-up, 20 were IAA and ICA positive, 7 had only IAA, 6 had only ICA (one of which was the only relative with restricted ICA who developed IDDM at age 68), and 1 relative was IAA and ICA negative.

57 normal individuals, 30 females and 27 males, were studied as a control group.

HLA typing. HLA-DR and -DQ typing was performed by serological methods. DQA1, DQB1, and DRB1 alleles were identified by DNA typing with a dot-blot technique using oligonucleotide sequence-specific probes (SSO). Two different protocols were used to ensure accuracy.

The first protocol and reagents were from the XI International HLA Workshop and were used for DQB1 typing (13). $1 \mu \mathrm{g}$ of DNA was amplified by PCR with specific primers. PCR conditions for the amplification of the second exon of the DQB1 gene were: 30 cycles, $94-96^{\circ} \mathrm{C}$ for $30 \mathrm{~s}, 56^{\circ} \mathrm{C}$ for $60 \mathrm{~s}$, and $72^{\circ} \mathrm{C}$ for $60-90 \mathrm{~s}$. PCR amplification products were spotted onto prewet nylon membranes (Hybond $\mathrm{N}$; Amersham Corp., Arlington Heights, IL) with a dot-blot apparatus (Bio-Rad Laboratories, Hercules, CA). After ultraviolet cross-linking $\left(0.12 \mathrm{~J} / \mathrm{cm}^{2}\right.$ for $\left.30 \mathrm{~s}\right)$, membranes were hybridized with ${ }^{32}$ P-labeled SSO. Each probe $(5-10 \mathrm{pmol})$ was labeled in a $25-\mu 1$ reaction with 60 $\mu \mathrm{Ci}$ of $\left[\gamma{ }^{32} \mathrm{P}\right] \mathrm{ATP}(\mathrm{sp}$ act $6,000 \mathrm{Ci} / \mathrm{mmol}$ ) in the presence of $20 \mathrm{U}$ of T4 polynucleotide kinase. Hybridization was performed in $50 \mathrm{mM}$ Tris- $\mathrm{HCl}$ ( $\mathrm{pH} 8.0$ ), $3 \mathrm{M}$ tetramethylammonium chloride (TMAC), 2 mM EDTA (pH 8.0), $5 \times$ Denhardt's solution, $0.1 \%$ SDS, and 100 $\mu \mathrm{g} / \mathrm{ml}$ heat-denatured salmon sperm DNA for $1-3 \mathrm{~h}$ at $54^{\circ} \mathrm{C}(18-\mathrm{mer}$ oligonucleotide probes). After hybridization, membranes were washed twice in $2 \times$ sodium chloride sodium phosphate EDTA buffer, $0.1 \%$ SDS at room temperature and twice in TMAC washing buffer $(50 \mathrm{mM}$ Tris- $\mathrm{HCl}$ [pH 8.0], $3 \mathrm{M}$ TMAC, 2 mM EDTA [pH 8.0], 0.1\% SDS) at $56-58^{\circ} \mathrm{C}$ (for 18 -mer oligonucleotide probes). Membranes were subjected to autoradiography by exposure to Kodak X-OMAT-AR films for $1-3 \mathrm{~h}$ at $-80^{\circ} \mathrm{C}$.

The second typing protocol used primers and horseradish peroxidase-labeled oligonucleotide probes developed by Cetus Corp. (Emeryville, CA), as described previously (14), for DQA 1, DQB1, and DRB1 typing. For some relatives, DQA1 alleles were typed using SSO probes

Table I. Clinical Characteristics of 82 at Risk First Degree Relatives

\begin{tabular}{lccccc}
\hline \multicolumn{1}{c}{ Relatives } & IAA+/ICA+ & IAA+/ICA- & IAA-/ICA+ & IAA-/ICA- & Total \\
\hline All & 40 & 32 & 9 & 1 & 82 \\
Prediabetic* & 20 & 7 & 6 & 1 & 34 \\
Nondiabetic & 20 & 25 & 3 & 0 & 48 \\
\hline
\end{tabular}

* Followed to the development of overt diabetes. developed by Cetus Corp. for forensic typing that are able to discriminate DQA 1 alleles differing at positions 40 and 51.

Statistical analysis. Chi square, Fisher's exact test, and rank sum test were used for statistical comparisons among the studied groups. Only two-tailed $P$ values are reported.

\section{Results}

The majority $(87.8 \%, 72 / 82)$ of first degree relatives at risk for diabetes had IAA levels $>39 \mathrm{nU} / \mathrm{ml}$. Extending our previous report $(8), 75 \%(54 / 72)$ of IAA-positive relatives carried a DR4 haplotype vs $20 \%(2 / 10)$ of IAA-negative relatives ( $P$ $=0.0004,54 / 72$ vs $2 / 10,95 \%$ confidence interval [C.I.] $=$ $0.02-0.4$, chi square). As shown in Fig. $1 A$, DR4-positive relatives had significantly higher IAA levels than DR4-negative relatives $($ mean $=370 \pm 70 \mathrm{SEM}$ vs mean $=198 \pm 84 \mathrm{SEM} \mathrm{nU} /$ $\mathrm{ml} ; P=0.001$, rank sum test). DR4 haplotypes carrying different DRB1 alleles $\left({ }^{*} 0401,{ }^{*} 0402,{ }^{*} 0404,{ }^{*} 0405\right)$ were equally associated with high IAA levels (data not shown).

18 IAA-positive individuals could be identified among 26 DR4-negative relatives. Table II shows their HLA-DR and -DQA1 alleles and their IAA levels ranging from 40 to 2,230 $\mathrm{nU} / \mathrm{ml}$. The observation that $25 \%(18 / 72)$ of IAA-positive relatives lacked DR4 indicated that DRB1 alleles on DR4 haplotypes do not account for all relatives with high IAA levels.

Since alleles at the DQ (DQA1 and DQB1 ) loci are significantly associated with diabetes susceptibility (15-19), we investigated potential associations between IAA levels and DQ alleles. With only one well-documented exception (19), the alleles found in our relatives did not differ from those predicted

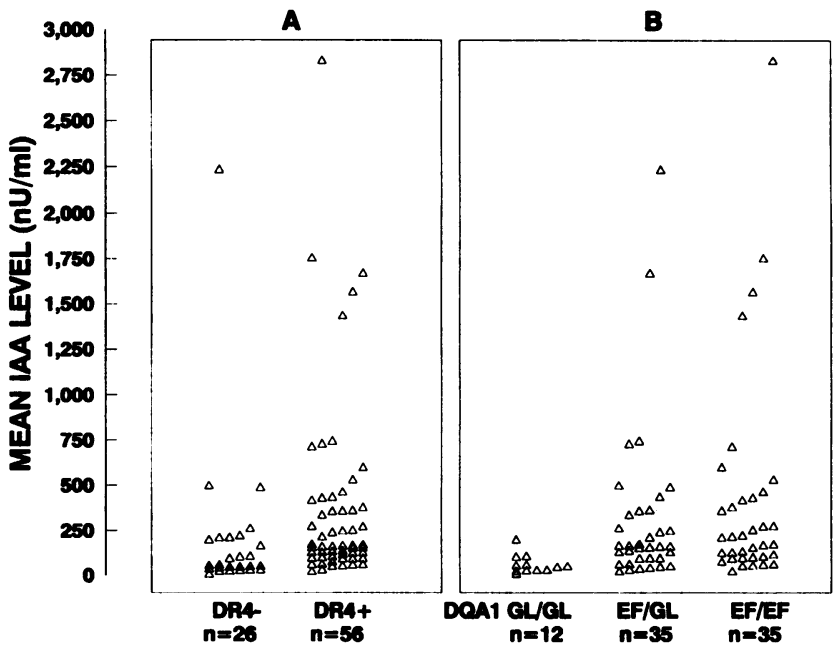

Figure 1. IAA levels in 82 first degree relatives at risk for diabetes subdivided by the presence/absence of DR4 and of DQA1 subtypes. (A) Most (75\%, 54/72) of IAA-positive relatives carried a DR4 haplotype. $98 \%(54 / 55)$ of DR4-positive relatives had IAA levels above normal with significantly higher IAA levels than DR4-negative relatives $($ mean $=370 \pm 70$ SEM vs mean $=198 \pm 84 \mathrm{SEM} \mathrm{nU} / \mathrm{ml} ; P$ $=0.001$, rank sum test). $(B)$ Most relatives $(70 / 82,85 \%)$ carried DQA 1 EF alleles (with no difference in the IAA level distribution between $35 \mathrm{EF} / \mathrm{EF}$ homozygotes and $35 \mathrm{EF} / \mathrm{GL}$ heterozygotes), and $93 \%(65 / 70)$ of them had IAA levels $>39 \mathrm{nU} / \mathrm{ml}($ mean $=360 \pm 63$ $\mathrm{SEM} \mathrm{nU} / \mathrm{ml}$ ). In contrast, among 12 relatives homozygous for $\mathrm{GL}$ alleles, only 7 had IAA levels above normal $(P=0.004,7 / 12$ vs $65 /$ $70,95 \%$ C.I. $=0.07-0.49$, Fisher's exact test; mean $=55 \pm 15$ SEM $\mathrm{nU} / \mathrm{ml}, P<0.0001$, rank sum test). Of note, $90 \%(65 / 72)$ of IAA-positive relatives had a DQA1 EF allele (including DR4-positive relatives), and only $75 \%(54 / 72)$ had a DR4 haplotype $(P=0.01)$. 
Table II. HLA-DR and -DQAI Alleles and IAA Levels for 18 DR4-negative Relatives with IAA Levels above Normal

\begin{tabular}{rrrrlll}
\hline Relative & Mean IAA & DR & DQA1 & $\begin{array}{c}\text { DQA1 allele } \\
\text { type }\end{array}$ & DQB1 \\
\hline & $n U / m l$ & & & & \\
1 & 40 & 3,3 & 0501,0501 & GL, GL & 0201,0201 \\
2 & 41 & 5,7 & 0501,0201 & GL, EF & 0301,0201 \\
3 & 43 & 3,8 & 0501,0401 & GL, GL & 0201,0402 \\
4 & 45 & 7,6 & 0201,0102 & EF, EF & 0201,0604 \\
5 & 49 & 3,3 & 0501,0501 & GL, GL & 0201,0201 \\
6 & 52 & 3,3 & 0501,0501 & GL, GL & 0201,0201 \\
7 & 88 & 3,1 & 0501,0101 & GL, EF & 0201,0501 \\
8 & 97 & 3,3 & 0501,0501 & GL, GL & 0201,0201 \\
9 & 101 & 3,5 & 0501,0501 & GL, GL & 0201,0301 \\
10 & 160 & 3,6 & 0501,0102 & GL, EF & 0201,0604 \\
11 & 193 & 3,3 & 0501,0501 & GL, GL & 0201,0201 \\
12 & 205 & 7,6 & 0201,0102 & EF, EF & 0201,0604 \\
13 & 206 & 3,9 & 0501,0301 & GL, EF & 0201,0303 \\
14 & 216 & 7,6 & 0201,0102 & EF, EF & 0201,0605 \\
15 & 256 & 3,2 & 0501,0102 & GL, EF & 0201,0502 \\
16 & 482 & 3,7 & 0501,0102 & GL, EF & 0201,0604 \\
17 & 492 & 3,2 & 0501,0102 & GL, EF & 0201,0602 \\
18 & 2230 & 3,1 & 0501,0101 & GL, EF & 0201,0501 \\
& & & & & \\
\hline
\end{tabular}

IAA levels are expressed as the mean of multiple measurements for each individual. Analysis of DQA 1 alleles indicates that 11 of the above relatives ( 7 of whom had the highest IAA levels) carried at least one DQA1 allele sharing sequences at positions 40 and 51 with DQA 1*0301 (see Fig. 2 for sequences).

by the linkage disequilibrium patterns among class II alleles (DR-DQ) in Caucasians (17).

Analysis of DQB1 subtypes among 56 DR4-positive at risk relatives revealed that 46 had the IDDM susceptibility allele
DQB $1{ }^{*} 0302(15,16)$ and 11 carried the neutral allele DQB $1 * 0301$ ( 8 relatives were DR4 homozygous and 2 were also DQB1 *0302/DQB1 *0301 heterozygous). However, the mean IAA level was not statistically different for these two groups of relatives $(\mathrm{DQB} 1 * 0302$ mean $=367 \pm 81 \mathrm{SEM} \mathrm{nU} /$ $\mathrm{ml}, n=46 ; \mathrm{DQB} 1{ }^{*} 0301$ mean $=505 \pm 166 \mathrm{SEM} \mathrm{nU} / \mathrm{ml}, n$ $=11, P=0.30, \mathrm{NS})$.

Since all Caucasian DR4 haplotypes carry the same allele at the DQA1 locus (DQA1 *0301), we compared the sequence of DQA ${ }^{*} 0301$ with DQA 1 alleles in the 18 IAA-positive relatives lacking a DR4 haplotype (Table II). Among the seven relatives with highest IAA levels in this group, one relative carried the same DQA $1{ }^{*} 0301$ allele usually linked to DR4 on a DR9 haplotype, and six relatives had a DQA1*01 allele (DQA $1 * 0102, n=5$; DQA1 ${ }^{*} 0101, n=1$ ). Considering the whole group of 18 relatives, DQA $1 * 01$ alleles were found in 9 of 18 relatives (DQA ${ }^{*} 0102, n=8$; DQA ${ }^{*} 0101, n=2$ ), and DQA $1{ }^{*} 0201$ (associated with DR7) was present in 4 relatives.

As Fig. 2 illustrates, all the above DQA1 alleles (DQA $1{ }^{*} 0101,{ }^{*} 0102,{ }^{*} 0103,{ }^{*} 0201,{ }^{*} 0301$ ) derive from evolutionary lineages 1,2 , and 3 and uniquely share glutamic acid (E) at position 40 and phenylalanine $(\mathrm{F})$ at position 51 (second exon) with the allele DQA1 *0301 found on DR4 haplotypes $(9,10)$. Therefore, we designate them DQA1 "EF" alleles. In contrast, DQA 1 alleles deriving from the evolutionary lineage $4\left(\mathrm{DQA} 1{ }^{*} 0401,{ }^{*} 0501\right.$, and *0601) usually not associated with high IAA levels carry glycine $(G)$ and leucine $(L)$ at positions 40 and 51 of the second exon (thus abbreviated as "GL" alleles).

As shown in Fig. $1 \mathrm{~B}, 70$ of 82 at risk relatives $(85 \%)$ carried at least one DQA1 EF allele, and 65 of these (93\%) had IAA levels $>39 \mathrm{nU} / \mathrm{ml}($ mean $=360 \pm 63$ SEM $\mathrm{nU} / \mathrm{ml})$. There was no difference in the mean IAA levels between EF/GL heterozygous ( mean $=300 \pm 74 \mathrm{SEM} \mathrm{nU} / \mathrm{ml}$ ) and $\mathrm{EF} / \mathrm{EF}$ homozygous relatives $($ mean $=422 \pm 102 \mathrm{SEM} \mathrm{nU} / \mathrm{ml})$. In contrast, only 7 of 12 relatives homozygous for DQA1 GL alleles (Table III)

EVOLUTIONARY LINEAGES 1, 2, and 3 (EF) ALLELES

ASSOCIATED DR Residue

10

51 DQA1*0101: DHVASCGNLYQFYGPSGQYTHEFDGDEEFYVDL \& RKETAWRWPE I SKFGGFDPQGALRNMAVAKHNLNIMIKRYNSTAATN DR1/DR6

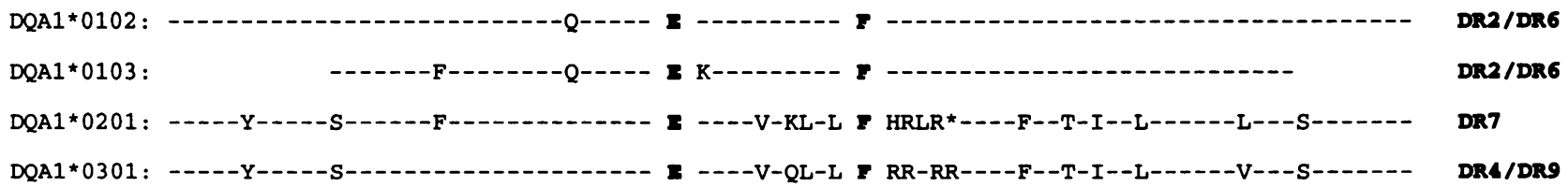

EVOLUTIONARY LINEAGE 4 (GL) ALLELES

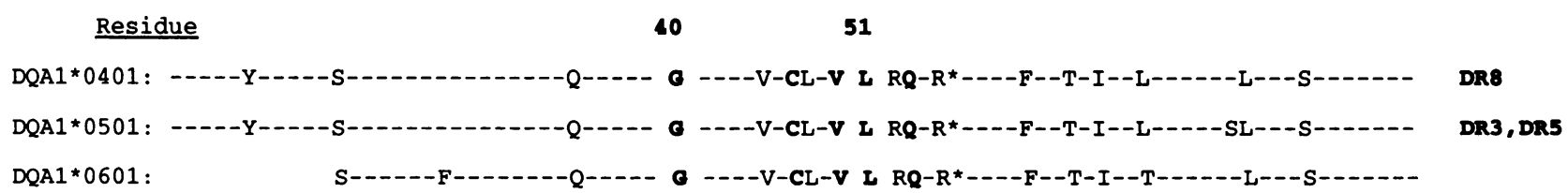

Figure 2. Amino acid sequences of second exon for DQA1 EF and GL alleles. With only one exception (11), DR, DQA, and DQB patterns found in our relatives did not differ from those predicted for Caucasians (12). Analysis of DQA1 amino acid sequences of the alleles reported in Table I, including DQA ${ }^{*} 0301$ allele found on DR4 haplotypes, showed that such alleles derive from the evolutionary lineages 1,2 , and 3 . These alleles share glutamic acid at position 40 and phenylalanine at position 51 . Therefore, they were named EF alleles. Three alleles derive from the evolutionary lineage 4. Since these alleles differ from EF alleles in that they carry glycine and leucine at positions 40 and 51 , they were named GL alleles. Position 51 on the DQ $\alpha$ chain is located in a putative peptide-binding site. Besides positions 40 and 51 , EF alleles differ from GL alleles also at positions 47,50 , and 53 , where GL alleles all carry cysteine, valine, and glutamine, respectively, at these residues. Thus, the region encompassed by amino acid residues $40-53$ is significantly different between the two subtypes of DQA1 alleles. 
Table III. DR, DQA1 Alleles and IAA Levels for Relatives Homozygous for DQA1 GL Alleles

\begin{tabular}{rrrrllll}
\hline Relative & $\begin{array}{c}\text { Mean } \\
\text { IAA }\end{array}$ & HLA-DR & DQA1 & $\begin{array}{c}\text { Allele } \\
\text { type }\end{array}$ & $\begin{array}{c}\text { Age } \\
\text { DM }\end{array}$ & Age \\
\hline & $n U / m l$ & & & & & & \\
1 & 1 & 3,8 & 0501,0401 & GL, GL & 23 & 38 \\
2 & 18 & 3,3 & 0501,0501 & GL, GL & 35 & 38 \\
3 & 20 & 3,3 & 0501,0501 & GL, GL & 50 & 53 \\
4 & 22 & 3,3 & 0501,0501 & GL, GL & - & 18 \\
5 & 24 & 3,5 & 0501,0501 & GL, GL & - & 14 \\
6 & 40 & 3,3 & 0501,0501 & GL, GL & - & 20 \\
7 & 43 & 3,8 & 0501,0401 & GL, GL & - & 31 \\
8 & 49 & 3,3 & 0501,0501 & GL, GL & 13 & 14 \\
9 & 52 & 3,3 & 0501,0501 & GL, GL & - & 41 \\
10 & 97 & 3,3 & 0501,0501 & GL, GL & 48 & 61 \\
11 & 101 & 3,5 & 0501,0501 & GL, GL & - & 25 \\
12 & 193 & 3,3 & 0501,0501 & GL, GL & 10 & 16 \\
& & & & & & \\
\hline
\end{tabular}

IAA levels are expressed as the mean of multiple measurements for each individual. All 12 relatives homozygous for GL alleles carried at least one DQA 1*0501 haplotype, and 8 out of 12 were DR3, DQA $1^{*} 0501$ homozygous. Among the four remaining relatives, two were DQA 1*0501 homozygous but DR3/DR5 heterozygous, and two were DQA1*0501/DQA1*0401 (DR3/DR8) heterozygous.

had IAA levels $>39 \mathrm{nU} / \mathrm{ml}(P=0.004,7 / 12$ vs $65 / 70,95 \%$ C.I. $=0.07-0.49$, Fisher's exact test; mean $=55 \pm 15$ SEM nU/ $\mathrm{ml}, P<0.0001$, rank sum test). Even excluding DR4-positive relatives from the calculation, those individuals with DQA1 EF alleles $(n=14)$ had remarkably higher levels than those ( $n$ $=12$ ) with GL alleles ( mean $=321 \pm 147 \mathrm{SEM} \mathrm{nU} / \mathrm{ml}$ vs mean $=55 \pm 15 \mathrm{SEM} \mathrm{nU} / \mathrm{ml}, P=0.02$, rank sum test). As shown in Fig. $1,90.2 \%(65 / 72)$ of IAA-positive relatives carried a DQA 1 EF allele (including DR4-positive relatives), while only $75 \%$ $(54 / 72)$ had a DR4 haplotype $(P=0.01,95 \%$ C.I. $=1.11-$ 8.89 , chi square). Moreover, only 5 of 10 (50\%) IAA-negative relatives had DQA1 EF alleles in contrast to the majority ( $90.2 \%)$ of IAA-positive relatives $(65 / 72, P=0.004,95 \%$ C.I. $=0.05-0.63$, Fisher's exact test). Although the frequency of DQA 1 EF alleles was not different between the 72 IAA-positive relatives and a group of 57 control subjects (data not shown), DQA1 EF alleles were found with a significantly lower fre-

Table IV. Mean Levels of IAA in 82 at Risk First Degree Relatives Subdivided by DQA1 Subtypes and the Three Criteria for at Risk Definition

\begin{tabular}{lccc}
\hline \multicolumn{1}{c}{ Relatives } & $\begin{array}{c}\text { DQA1 EF/X* } \\
\text { IAA levels }\end{array}$ & $\begin{array}{c}\text { DQA1 GL/GL } \\
\text { IAA levels }\end{array}$ & $\begin{array}{c}\text { Rank } \\
\text { sum test }\end{array}$ \\
\hline & mean $\pm S E M$ & \multicolumn{1}{c}{ mean $\pm S E M$} & \\
Prediabetic $^{\ddagger}$ & $265 \pm 60(24 / 27)^{8}$ & $63 \pm 27(4 / 7)^{8}$ & $P=0.01$ \\
ICA-positive $^{8}$ & $333 \pm 81(34 / 39)^{8}$ & $60 \pm 16(6 / 10)^{8}$ & $P=0.001$ \\
ICA-/IAA+ & $393 \pm 98(31 / 31)^{8}$ & $52(1 / 1)^{8}$ &
\end{tabular}

Criteria are detailed in Methods. ${ }^{*} \mathrm{X}=\mathrm{EF}$ or GL. ${ }^{\ddagger}$ Followed to the development of overt diabetes. ${ }^{8}$ Number of IAA+/total number. Note that categories are not all mutually exclusive. For example, of the 34 prediabetics, 26 were ICA positive, and 8 were ICA negative. quency among IAA-negative relatives $(50 \%, 5 / 10)$ than in controls $(92.9 \%, 53 / 57 ; P=0.002,95 \%$ C.I. $=-0.8--0.14$, Fisher's exact test).

To more precisely evaluate the correlation of DQA1 subtypes with IAA levels, we subdivided our relatives into three not mutually exclusive groups according to the criteria used for the at risk definition detailed in Methods. As Table IV illustrates, the mean IAA level of relatives with DQA1 EF alleles was significantly higher than that of relatives homozygous for DQA 1 GL alleles in all groups considered (relatives followed to overt IDDM, ICA-positive, and IAA-positive relatives).

In that IAA levels are inversely associated with age of diabetes diagnosis (6), we plotted the IAA levels of our 82 relatives, subdividing them by DQA1 subtypes and age (Fig. 3). The markedly lower levels of IAA in those relatives homozygous for DQA1 GL alleles are apparent, consistent with the hypothesis that DQA1 GL alleles (or any gene on the haplotypes carrying such alleles) confer recessive low responsiveness to insulin.

Although the prevalence of diabetes was not significantly different in relatives with DQA 1 EF or GL alleles (36 vs $50 \%$ ), the mean age of diabetes diagnosis was somewhat higher in relatives with DQA1 GL alleles ( mean age $=29.9 \pm 4.6 \mathrm{SEM}$ yr) than in those with DQA1 EF alleles (mean age $=18.3 \pm 3.2$ SEM yr, $P=0.07$, rank sum test).

\section{Discussion}

Among other autoantibodies detectable in the serum of prediabetics or newly diagnosed patients, IAA precede IDDM onset and correlate with rate of progression to diabetes (1-5). IAA levels are usually stable during the prediabetic period $(5,6)$, suggesting that they may be genetically determined. Although DR4 haplotypes are strongly associated with higher levels of IAA (8), these haplotypes were not found in all relatives with high IAA levels since $18 / 26(69.2 \%)$ DR4-negative relatives also had IAA levels exceeding our normal range of $39 \mathrm{nU} / \mathrm{ml}$ (Fig. $1 B$ and Table II). Based on this observation and on our analysis of DR4 DRB1 subtypes (we could observe no difference in IAA levels among DRB1 subtypes), we suspected that DRB1 alleles on DR4 haplotypes were not primarily responsible for elevated IAA levels. Instead, our data suggested the hypothesis that genes other than DRB1 alleles on DR4 haplotypes were responsible for the association with high IAA levels and that such alleles should be common to DR4 and non-DR4 haplotypes. A candidate to improve the correlation with IAA levels was the HLA-DQ loci. These loci have been closely associated with susceptibility (15-19) and protection (18) from IDDM and are close to the DRB1 locus on chromosome 6.

Therefore, to further investigate the genetic determinants of IAA, we HLA-typed 82 at risk first degree relatives of IDDM patients (of whom 72 were IAA positive) using PCR/SSO methods for DQA1 and DQB1 alleles. Analysis of DQB1 subtypes did not show any better association with IAA than DR4 positivity, and there was no statistical difference between the IAA levels found in individuals with DQB ${ }^{*} 0302$ or DQB1 *0301 among our DR4-positive relatives.

All DR4 haplotypes carry the same allele at the DQA 1 locus (DQA ${ }^{*} 0301$ ). Analysis of DQA1 alleles in those 18 IAA-positive relatives lacking a DR4 haplotype ( Table II) showed that 11 individuals (among whom were 7 with the highest IAA levels) carried at least one DQA 1 allele sharing sequences at posi- 


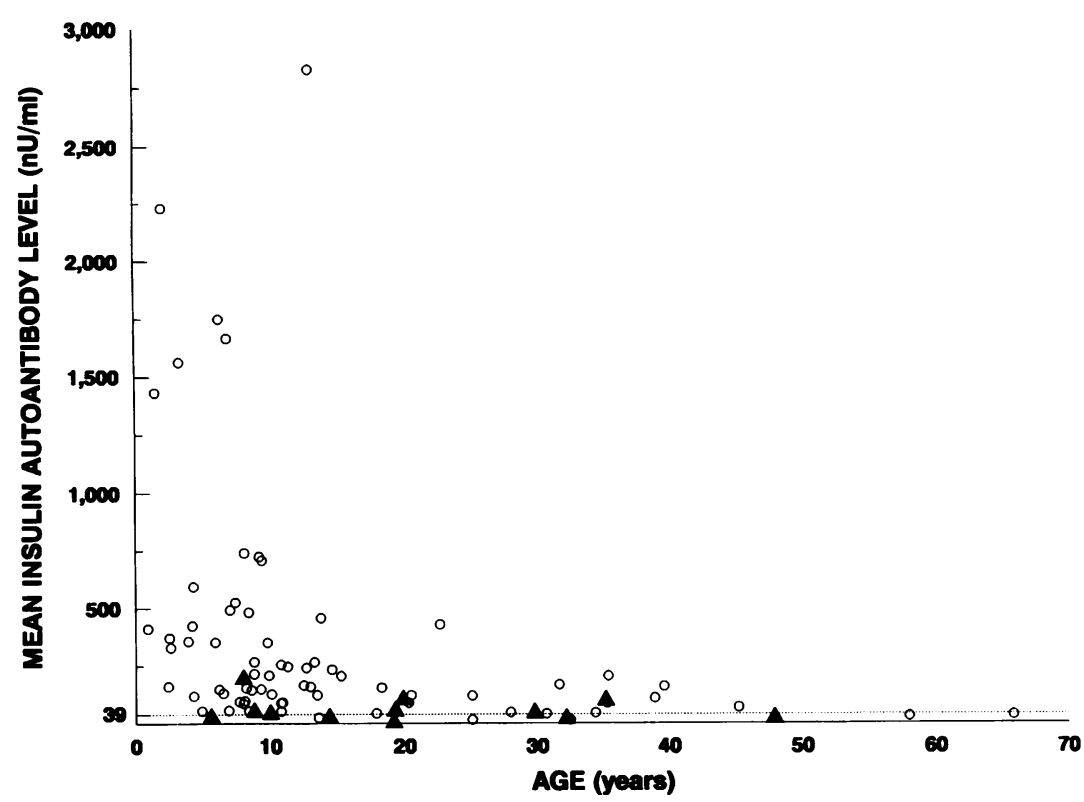

Figure 3. Mean IAA levels versus age for relatives with DQA1 EF or DQA1 GL alleles. Relatives with DQA1 GL (solid triangles) alleles are characterized by low IAA levels and do not show a clear influence of age on IAA levels. In contrast, relatives with DQA1 EF alleles (open circles) have higher IAA levels particularly apparent in children. tions 40 and 51 with DQA ${ }^{*} 0301\left({ }^{*} 0101, *^{*} 0102,{ }^{*} 0201\right.$, $\left.{ }^{*} 0301\right)$. As shown in Fig. 2, analysis of the amino acid sequences of the above DQA 1 alleles revealed that all alleles were from the evolutionary lineages $1-3(9,10)$ and share glutamic acid $(E)$ at position 40 and phenylalanine $(F)$ at position 51 (EF alleles: DQA $1{ }^{*} 0101,{ }^{*} 0102,{ }^{*} 0103,{ }^{*} 0201,{ }^{*} 0301$ ). DQA1 EF alleles are found on DR1, DR2, DR4, DR6, and DR7 haplotypes (Fig. 2). Most relatives (70/82, 85\%) carried at least one DQA1 EF allele, and the great majority of EF relatives (93\%, $65 / 70$ ) had IAA levels above normal (mean $=360 \pm 63$ SEM $\mathrm{nU} / \mathrm{ml})$. Most of our IAA-positive relatives $(90 \%, 65 / 72)$ carried a DQA1 EF allele, while only $75 \%(54 / 72)$ of them were DR4 positive ( $P=0.01$, Fig. 1$)$. Of note, $5 / 70$ relatives with a DQA1 EF allele lacked IAA, suggesting that other genes or environmental factors may influence the appearance of IAA.

In contrast to DQA 1 EF alleles, other DQA1 alleles (GL alleles: DQA $1{ }^{*} 0401,{ }^{*} 0501,{ }^{*} 0601$ ) were not associated with high IAA levels and have glycine and leucine at positions 40 and 51 of the second exon (Fig. 2). As Table III illustrates, only 7 of 12 relatives homozygous for GL alleles were IAA positive ( $P=0.004,7 / 12$ vs $65 / 70$ relatives with EF alleles ), and only 2 of them had IAA levels $>100 \mathrm{nU} / \mathrm{ml}$. The mean IAA level for this group was significantly lower than in relatives with EF alleles $($ mean $=55 \pm 15 \mathrm{SEM} \mathrm{nU} / \mathrm{ml}$ vs mean $=360 \pm 63 \mathrm{SEM}$ $\mathrm{nU} / \mathrm{ml}, P<0.0001$ ). Therefore, homozygosity for DQA1 GL alleles appears to be associated with a "recessive" lack of humoral response to insulin. Moreover, relatives homozygous for DQA1 GL alleles had lower levels of IAA at all ages in contrast to relatives with DQA1 EF alleles (Fig. 3). The two DQA1 subtypes showed a similar correlation with IAA levels, even when we subdivided our at risk relatives according to the individual criteria used for entering the study (Table IV). Although the frequency of DQA1 EF alleles was not different between IAA-positive relatives and normal controls, DQA 1 EF alleles were found with a significantly lower frequency among IAA-negative relatives $(50 \%, 5 / 10)$ than in controls $(92.9 \%$, $53 / 57 ; P=0.002$ ), and conversely DQA1 GL alleles have a higher frequency among IAA-negative relatives.

Among DQA1 GL alleles, DQA ${ }^{*} 0601$ is not found in Caucasians, and DQA $1{ }^{*} 0401$ is carried only on rare DR8 hap- lotypes. As a consequence, the great majority of GL alleles in our relatives is represented by DQA 1 *0501 found on DR3 haplotypes. Indeed ( Table III), all 12 relatives homozygous for GL alleles carried a DR3, DQA 1 *0501 haplotype, and 8 out of 12 were DR3, DQA1*0501 homozygous. However, among the four remaining relatives, two were $\mathrm{DQA} 1{ }^{*} 0501$ homozygous but DR3/DR5 heterozygous, and two were DQA1*0501/ DQA 1 *0401 (DR3/DR8) heterozygous.

A similar association of low levels of insulin antibodies with DR3 homozygosity has been reported previously (20) following insulin administration after diabetes onset. In agreement with the above study in IDDM patients, our findings in relatives at risk for diabetes support the hypothesis that DR 3 haplotypes contain genes associated with a lack of humoral immune response to endogenous insulin and suggest an association with DQA 1 sequences. The finding that a significant portion $(4 / 12$, $33 \%$ ) of relatives homozygous for DQA1 GL alleles carried such alleles also on DR5 (DQA1*0501) and DR8 (DQA $1 * 0401$ ) haplotypes supports the hypothesis that DQA1 GL alleles (and not only DQA1 *0501, DR3 haplotypes) are associated with a lack of humoral response to insulin.

Of interest, as indicated by the recently reported three-dimensional structure of a class II molecule (21), position 51 on the $\alpha$ chain lies within the peptide-binding site. Besides positions 40 and 51, DQA1 EF alleles differ from GL alleles also at positions 47,50 , and 53 , where GL alleles all have cysteine, valine, and glutamine, respectively, at these residues (Fig. 2). Thus, the region encompassed by amino acid residues $40-53$ is dramatically different between these two DQA1 subtypes, and we hypothesize that this may influence binding and presentation of insulin peptides. A similar association has been described recently between anticentromere autoantibodies and polar amino acids at position 26 of the HLA-DQB1 first domain (second exon) in scleroderma (22).

In this study, relatives with DQA 1 GL alleles developed diabetes at an older age (mean age $=29.9 \pm 4.6 \mathrm{SEM}$ yr) than those with DQA1 EF alleles (mean age $=18.3 \pm 3.2 \mathrm{SEM}$ yr, $P$ $=0.07$, rank sum test), confirming previous reports that the lack of immune response to insulin may be associated with a slower rate of the diabetogenic process $(3,6)$. 
In conclusion, once tolerance to insulin is broken during the prediabetic phase, DQA 1 EF alleles show the strongest association (stronger than DR4) with prevalence and higher levels of IAA. Further analysis is required to address the question of whether this association is the consequence of the recessive lack of response mediated by DQA1 GL alleles (and DR3, DQA $1{ }^{*} 0501$ homozygosity in particular). The differences in the DQA 1 amino acid sequences discussed above may be associated with the phenotypic variation observed and support the hypothesis of two functional DQA1 allele subsets which correlate with different levels of the humoral immune response to insulin. If confirmed by further studies, DQA1 typing may improve our ability to predict the humoral immune response to insulin and may explain a major form of phenotypic variation among prediabetic first degree relatives of patients with type I diabetes.

\section{Acknowledgments}

We thank Sunanda Babu for excellent technical support, Terry Smith for contributing her nursing skills, and Dennis Dunlop for secretarial help. We are also grateful to Dr. David Talmage for his critical suggestions on reading the manuscript.

This study was supported by National Institutes of Health grants DK-32083, DK-39233, DK-33790, DK-26844, DK-29583, and DK35449; The Juvenile Diabetes Foundation; The American Diabetes Association; The Greenwall Foundation; and the Blum Kovler Foundation. The Joslin Diabetes Center DERC (DK-36836) clinical core was essential for the described studies. A. Pugliese is a recipient of a research fellowship from the Juvenile Diabetes Foundation.

\section{References}

1. Pugliese, A., and G. S. Eisenbarth. 1992. Type I diabetes mellitus: lessons for human autoimmunity. J. Lab. Clin. Med. 120:363-366.

2. Atkinson, M. A., and N. K. Maclaren. 1990. What causes diabetes? Sci. Am. 263:62-71.

3. Rotter, J. I., C. M. Vadheim, and D. L. Rimoin. 1992. Diabetes Mellitus. In The Genetic Basis of Common Diseases. R. A. King, J. I. Rotter, and A. G. Motulsky, editors. Oxford University Press, New York, Oxford. 413-481.

4. Palmer, J. P., C. M. Asplin, P. Clemons, K. Lyen, O. Tatpati, P. K. Raghu, and T. L. Paquette. 1983. Insulin antibodies in insulin-dependent diabetics before insulin treatment. Science (Wash. DC). 222:1337-1339.

5. Eisenbarth, G. S., R. A. Jackson, and A. Pugliese. 1992. Insulin autoimmunity: the rate limiting factor in pre-type I diabetes? J. Autoimmun. 5:241-246.

6. Vardi, P., S. A. Dib, M. Tuttleman, J. E. Connelly, M. Grinbergs, A. Rabizadeh, W. J. Riley, N. K. Maclaren, G. S. Eisenbarth, and J. S. Soeldner.
1987. Competitive insulin autoantibody RIA: prospective evaluation of subjects at high risk for development of type I diabetes mellitus. Diabetes. 36:1286-1291.

7. Ziegler, A. G., R. Ziegler, P. Vardi, R. A. Jackson, J. S. Soeldner, and G. S. Eisenbarth. 1989. Life-table analysis of progression to diabetes of anti-insulin autoantibody positive relatives of individuals with type I diabetes. Diabetes. 38:1320-1325

8. Ziegler, R., C. A. Alper, Z. L. Awdeh, L. Castano, S. J. Brink, J. S. Soeldner, R. A. Jackson, and G. S. Eisenbarth. 1991. Specific association of HLA-DR4 with increased prevalence and level of insulin autoantibodies in first degree relatives of patients with type I diabetes. Diabetes. 40:709-714.

9. Gyllensten, U. B., and H. A. Erlich. 1989. Ancient roots for polymorphism at the HLA-DQ alpha locus in primates. Proc. Natl. Acad. Sci. USA. 86:99869990.

10. Erlich, H. A., and U. B. Gyllensten. 1991. The evolution of allelic diversity at the primate major histocompatibility complex class II loci. Hum. Immunol. 30:110-118.

11. Srikanta, S., A. Rabizadeh, M. A. K. Omar, and G. S. Eisenbarth. 1985. Assay for islet cell antibodies: protein A-monoclonal antibody method. Diabetes. 34:300-305

12. Gianani, R., A. Pugliese, S. Bonner-Weir, A. J. Shiffrin, J. S. Soeldner, H. Erlich, Z. L. Awdeh, C. A. Alper, R. A. Jackson, and G. S. Eisenbarth. 1992. Prognostically significant heterogeneity of cytoplasmic islet cell antibodies in relatives of patients with type I diabetes. Diabetes. 41:347-353.

13. Ronningen, K. S., A. Spurkland, B. D. Tait, B. Drummond, C. LopezLarrea, F. S. Baranda, M. J. Menendez-Diaz, S. Caillat-Zucman, G. Beaurain, H. J. Garchon, et al. 1991. HLA class II associations in insulin-dependent diabetes mellitus among Blacks, Caucasoids, and Japanese. In HLA 1991. K. Tsuji, M. Aizawa, and T. Sasazuki, editors. Oxford University Press, Oxford/New York/Tokyo. 713-722.

14. Saiki, R. K., T. L. Bugawan, G. T. Horn, K. B. Mullis, and H. A. Erlich. 1986. Analysis of enzymatically amplified $\beta$-globin and HLA-DQ $\alpha$ DNA with allele specific oligonucleotide probes. Nature (Lond.). 324:163-166.

15. Todd, J. A., J. I. Bell, and H. O. McDevitt. 1987. HLA-DQ beta gene contribution to susceptibility and resistance to insulin dependent diabetes mellitus. Nature (Lond.). 329:599-604.

16. Horn, G. T., T. L. Bugawan, C. M. Long, and H. A. Erlich. 1988. Allelic sequence variation of the HLA-DQ loci: relationship to serology and to insulindependent diabetes susceptibility. Proc. Natl. Acad. Sci. USA. 85:6012-6016.

17. Nepom, G. T., and H. Erlich. 1991. MHC class-II molecules and autoimmunity. Annu. Rev. Immunol. 9:493-525.

18. Baisch, J. M., T. Weeks, R. Giles, M. Hoover, P. Stastny, and J. D. Capra. 1990. Analysis of HLA-DQ genotypes and susceptibility in insulin-dependent diabetes mellitus. $N$. Eng. J. Med. 322:1836-1841.

19. Erlich, H. A., R. L. Griffith, T. L. Bugawan, R. Ziegler, C. Alper, and G. S. Eisenbarth. 1991. Implication of specific DQB1 alleles in genetic susceptibility and resistance by identification of IDDM siblings with novel HLA-DQB 1 allele and unusual DR1 and DR1 haplotypes. Diabetes. 40:478-481.

20. McEvoy, R. C., M. E. Witt, F. Ginsberg-Fellner, and P. Rubinstein. 1986. Anti-insulin antibodies in children with type I diabetes mellitus. Diabetes. 35:634-641.

21. Brown, J. H., T. Jardetzky, J. C. Gorga, L. J. Stern, R. G. Urban, J. L. Strominger, and D. C. Wiley. 1993. Three-dimensional structure of the human class II histocompatibility antigen HLA-DR1. Nature (Lond.). 364:33-39.

22. Reveille, J. D., D. Owerbach, R. Goldstein, R. Moreda, R. A. Isern, and F. C. Arnett. 1992. Association of polar amino acids at position 26 of the HLADQB1 first domain with the anticentromere autoantibody response in systemic sclerosis (scleroderma). J. Clin. Invest. 89:1208-1213. 